\title{
Use of Oral Agents and/or Insulin in the Treatment of Diabetes during Pregnancy: An Examination of Outcomes in Pregestational versus Gestational Diabetics
}

\author{
Edwin Henslee, Amanda Hatton, Selman I. Welt, Jennifer Holmes, Lindsay Penrose, \\ Samuel Prien, Naghma Farooqi \\ Department of Obstetrics and Gynecology, Texas Tech University Health Sciences Center, Lubbock, USA \\ Email: naghma.faroogi@ttuhsc.edu
}

Received 27 September 2014; 25 October 2014; accepted 21 November 2014

Academic Editor: Christos E. Constantinou, Stanford University Medical School, USA

Copyright (C) 2014 by authors and Scientific Research Publishing Inc.

This work is licensed under the Creative Commons Attribution International License (CC BY). http://creativecommons.org/licenses/by/4.0/

(c) (i) Open Access

\begin{abstract}
The management of diabetes in pregnancy varies depending on whether the condition was first diagnosed during pregnancy (gestational diabetes) or was diagnosed before pregnancy (pregestational diabetes). Little has been published comparing the relative efficacy of various oral agents for the treatment of gestational diabetes and the reported experience with the insulin pump in pregnancy for pregestational diabetes remains meager. We conducted a retrospective chart review of women managed in a specialized diabetic clinic to compare the results of treatment of gestational diabetes with oral agents, glyburide and acarbose, to those treated with split-mixed insulin and treatment of pregestational diabetes with either the insulin pump or conventional splitmixed insulin. Gestational diabetics treated with split-mixed insulin were hospitalized significantly more often $(p<0.001)$ than those treated with oral agents only. The incidence of several important pregnancy complications (growth restriction, preterm labor, preeclampsia, oligohydramnios) did not differ between groups. Pregestational diabetics managed with an insulin pump had comparable glycemic control, as measured by hemoglobin A1c, to those managed with split-mixed insulin. Infant birth weights and Apgar scores were similar in each group. There were no perinatal deaths in either group. Acarbose and glyburide showed comparable efficacy in treating gestational diabetics. In addition, our experience adds to the small number of pregnant women with pregestational diabetes who were managed with an insulin pump that have been reported in the literature.
\end{abstract}

\section{Keywords}

Diabetes, Pregnancy, Treatment Methods, Pump, Oral Agents 


\section{Introduction}

Health care personnel in the United States today are struggling to contend with two interrelated epidemics: Obesity and diabetes [1] [2]. Those who provide care for pregnant women are challenged by not only obesity and diabetes, but by the need to optimize pregnancy outcome for both women and their offspring. In response to this challenge, many institutions have established specialized antenatal clinics specifically to provide care to pregnant diabetics, including women with gestational diabetes and those with pregestational diabetes.

At Texas Tech University Health Sciences Center in Lubbock, Texas, we established a half-day Diabetic Clinic in 2005. Since then we have seen the census grow steadily, reflecting the epidemic noted above, and it is apparent that a full day will be required in the near future. Turnover in providers, staff, and department leadership has made it difficult to maintain a consistent approach to management of diabetes, and the obvious differences in the treatment of gestational and pregestational diabetes adds a layer of complexity.

Prior to the Year 2000, women with gestational diabetes who did not respond to diet were almost universally treated with insulin. The report by Langer and colleagues [3] on the efficacy of glyburide immediately altered that approach. Glyburide orally was clearly preferable to women not previously diagnosed with diabetes compared to the alternative of 2 or 3 subcutaneous injections daily. Glyburide was effective at lowering mild fasting hyperglycemia. Another oral agent, acarbose, was found to lower postprandial hyperglycemia, although the evidence base was much less compelling for either safety or efficacy [4]. It was decided to try one or the other or both to manage class A2 gestational diabetes in our clinic before resorting to insulin treatment.

For the management of pregestational diabetes, most centers reserved insulin pump use for women who failed treatment with the more conventional split-mixed approach. At least two factors led us to adopt an alternate strategy: First, several faculty members had previous favorable experience with insulin pumps and second, our diabetic nurse devoted a great deal of time explaining the use and advantages of the pump to suitable candidates. Therefore, women who were highly motivated and knowledgeable about their disease were offered the insulin pump as primary therapy.

The purpose of this report is to describe the management of pregnant diabetics from our diabetic clinic. We partitioned the patients into two groups: Gestational diabetics that could not be controlled on diet alone and pregestational diabetics managed either with continuous subcutaneous insulin infusion (CSII) by insulin pump or with traditional split-mixed insulin. The group with gestational diabetes was subdivided into those requiring only oral therapy and those requiring split-mixed insulin. Lastly, the oral therapy group was analyzed according to the type of oral treatment: Glyburide alone, acarbose alone, or both agents in combination.

\section{Materials and Methods}

We conducted a retrospective chart review of 245 women managed in our diabetic clinic at Texas Tech University Health Sciences Center from 2005 to 2009. One team of investigators focused on women with gestational diabetes managed from 2005 to 2008, while a second team focused on pregestational diabetics cared for between 2006 and 2009. For each arm of the study, approval was obtained from the institutional review board. The data from each arm were then pooled for presentation at the District XI meeting of the American College of Obstetricians and Gynecologists (ACOG) in October 2010. Inclusion criteria for the gestational diabetes arm were age 18 - 40, prenatal care provided in our clinic and delivery at our teaching hospital. Women were diagnosed with gestational diabetes if they had $\geq 2$ abnormal values on a 3-hour oral glucose tolerance test according to the criteria of Coustan and Carpenter [5] or if they had a one hour glucose challenge test result of $>200 \mathrm{mg} / \mathrm{dl}$. All women received detailed nutritional counseling by a registered dietitian at entry to care and as needed throughout the prenatal course. Women were considered to have pregestational diabetes only if that diagnosis was documented in the medical record prior to the index pregnancy. Charts reviewed by both teams included antenatal clinic records, maternal hospital records and neonatal records.

Hemoglobin A1c levels were obtained at entry to care and repeated later in the pregnancy at a nonstandardized gestational age. Demographic data including age, gestational age at entry to care and at delivery, parity and number of prenatal visits were abstracted for all women, along with class of diabetes and all forms of treatment. Maternal weight was recorded at entry to care and again at delivery. Infant birth weight, Apgar scores and any maternal or perinatal complications were noted. Women with multifetal pregnancies, other preexisting medical conditions (e.g., lupus, thyroid disease, etc.), late (>35 weeks EGA) diagnosis, and inadequate prenatal care ( $>2$ missed appointments) were excluded from analysis. 
Patients in both arms were grouped by diabetes treatment as documented in their blood sugar log book. For the pregestational diabetics, treatment groups consisted of either split-mixed insulin or the insulin pump. The decision to place a patient on an insulin pump was dependent on whether the patient was willing and able to learn how to operate and manage the pump. Patients that were started on split-mixed insulin received a weightbased dose of a short acting (regular) and an intermediate acting insulin (N) in four daily doses. Patients placed on the insulin pump received a short acting insulin (regular). A variable basal rate was set with prandial boluses dependent on carbohydrate intake and caloric distribution.

For the gestational diabetics, treatment groups consisted of a split-mixed injectable insulin or oral agents. The decision to place a patient on insulin versus an oral agent was made after reviewing the patient's fingerstick blood sugar logbook. If $>50 \%$ of 1-hour postprandial glucose values were $>200 \mathrm{mg} / \mathrm{dl}$, the patient was started on insulin without a trial of oral agents. For those with $<50 \%$ of postprandial values $>200 \mathrm{mg} / \mathrm{dl}$, treatment with an oral agent was initiated. If the patient's fasting blood sugar was elevated, she was started on glyburide. If her postprandial fingerstick blood sugars were elevated, then acarbose was started. As in the pregestational group, patients on split-mixed insulin received a weight based dose of a short acting (regular) and an intermediate acting insulin $(\mathrm{N})$ in four daily doses.

Data were analyzed using the Statistical Package for the Social Sciences (Version 12). Student's t-test was used to compare means for continuous data and the chi square test was used for nominal scale data. A p-value of $<0.05$ was considered statistically significant. ANOVA testing and Tukey's mean separation test were used to compare results for the three groups of gestational diabetics treated with oral agents.

\section{Results}

For the pregestational diabetics, demographic information is displayed in Table 1 . There were no significant differences in age or parity, but women treated with the insulin pump began their prenatal care significantly earlier than women in the split-mixed group. Insulin pump patients also had more prenatal visits. Shown in Table 2 are data pertaining to maternal weight at delivery, initial and repeat hemoglobin A1c, birth weight, and Apgar scores.

There was no difference between groups in the following pregnancy complications: Pre-eclampsia, dystocia, premature rupture of membranes, preterm delivery and polyhydramnios. For newborns, the admission rate to the neonatal intensive care unit likewise did not differ between groups.

Patient demographic information for gestational diabetics was comparable for women treated with oral agents $(\mathrm{N}=123)$ to those treated with split-mixed insulin $(\mathrm{N}=57)$. Mean infant birth weight for the group treated with oral agents was 3199 grams, compared to 3185 grams for the split-mixed group. One and 5 minute Apgar scores were similar between groups. Table 3 contains data comparing treatment with oral agents to treatment with split-mixed insulin for women with gestational diabetes. Women treated with split-mixed insulin were hospitalized much more frequently than women treated with oral agents. In Table 4 the three oral agent groups were

Table 1. Demographic data for pregestational diabetics.

\begin{tabular}{cccc}
\hline & Insulin Pump $(\mathrm{N}=33)$ & Split-Mixed Insulin $(\mathrm{N}=29)$ & p-Value \\
\hline Maternal age & 26.1 & 28.6 & $<0.05$ \\
EGA at entry & 11.0 & 16.0 & $<0.02$ \\
$\begin{array}{c}\text { (weeks) } \\
\text { EGA at delivery } \\
\text { (weeks) }\end{array}$ & 37.3 & & 0.27 \\
$\begin{array}{c}\text { Prenatal visits } \\
3\end{array}$ & 0 & 36.3 & \\
$4-7$ & 1 & 3 & \\
$8-11$ & 6 & 6 & \\
$\geq 12$ & 26 & 9 & \\
\hline
\end{tabular}


Table 2. Outcomes for pregestational diabetics.

\begin{tabular}{cccc}
\hline & Insulin Pump (N = 33) & Split-Mixed Insulin $(\mathrm{N}=29)$ & $\mathrm{p}$-Value \\
\hline Maternal weight at entry & $82.3 \mathrm{~kg}$ & $95.6 \mathrm{~kg}$ & $<0.05$ \\
Maternal weight at delivery & $94.7 \mathrm{~kg}$ & $103.7 \mathrm{~kg}$ & 0.17 \\
Hemoglobin A1c at entry & $8.6 \%$ & $8.1 \%$ & 0.36 \\
Hemoglobin A1c after treatment & $6.7 \%$ & $6.6 \%$ & 0.77 \\
Birth weight & $3353.4 \mathrm{~g}$ & $3245.0 \mathrm{~g}$ & 0.62 \\
Apgar (1 minute) & 7 & 7 & 0.66 \\
Apgar (5 minute) & 8 & 8 & 0.54 \\
\hline
\end{tabular}

Table 3. Complications in women with gestational diabetes stratified by method of treatment.

\begin{tabular}{cccc}
\hline & Oral Agents $(\mathrm{N}=123)$ & Split-Mixed Insulin $(\mathrm{N}=57)$ & $\mathrm{p}$-Value \\
\hline Hospitalizations & $14 \%$ & $64 \%$ & $<0.001$ \\
IUGR & $4 \%$ & $3.6 \%$ & 0.91 \\
Oligohydramnios & $21.6 \%$ & $21.8 \%$ & 0.97 \\
Preeclampsia & $4 \%$ & $7.3 \%$ & 0.35 \\
Preterm delivery & $22 \%$ & $24.6 \%$ & 0.56 \\
\hline
\end{tabular}

Table 4. Outcomes for patients treated with oral agents.

\begin{tabular}{ccccc}
\hline & Acarbose $(\mathrm{N}-67)$ & Glyburide $(\mathrm{N}=37)$ & ${\text { Combination }(\mathrm{N}=19)^{*}}^{\mathrm{p} \text {-Value }}$ \\
\hline Hospitalizations & $9 \%$ & $24.3 \%$ & $15.8 \%$ & 0.10 \\
Hypoglycemia & $14.9 \%$ & $24.3 \%$ & $10.5 \%$ & 0.34 \\
IUGR & $3 \%$ & $0 \%$ & $5.3 \%$ & 0.44 \\
Oligohydramnios & $20.9 \%$ & $21.6 \%$ & $26.3 \%$ & 0.88 \\
Preeclampsia & $1.5 \%$ & $2.7 \%$ & $15.8 \%$ & 0.02 \\
Preterm delivery & $17.9 \%$ & $29.7 \%$ & $21.2 \%$ & 0.35 \\
Respiratory distress & $7.5 \%$ & $18.9 \%$ & $0 \%$ & 0.05 \\
Hyperbilirubinemia & $1.5 \%$ & $8.1 \%$ & $0 \%$ & 0.13 \\
\hline
\end{tabular}

compared with regard to a number of variables. Patients taking either acarbose, glyburide, or a combination of the two had similar rates of hospitalizations and hypoglycemic events ( $p=0.338)$, and similar rates of complications during pregnancy (Table $4 ; \mathrm{p}=0.376$ ). Preeclampsia rates were significantly higher in those patients receiving the combined therapy ( $\mathrm{p}<0.02)$, however there was no explanation for this finding based on available chart information. Neonates had similar rates of NICU admissions ( $p=0.196)$ and rates of hyperbilirubinemia ( $p$ $=0.131$ ). However, there appeared to be a significantly higher rate of respiratory complications in those infants whose mother received glyburide.

\section{Discussion}

There are substantial benefits to both patients and providers as a result of having a specialized antenatal diabetic clinic. Standardized management is more readily achieved when the same personnel (faculty, nurse educator, nutritionist) see the same group of patients regularly, frequently, and longitudinally. It is widely appreciated that the management of pregnant diabetics involves more than the use of insulin or oral hypoglycemic agents. Diet, 
exercise, faithful recording of fingerstick blood glucose values, and compliance with prenatal visits all affect outcome as much or more than the actual medications administered. Health care providers in the diabetic clinic form close relationships with the patients, fostering compliance and facilitating the monitoring of glycemic control. "Tight control" or "rigid control" of blood glucose is not always achievable in the individual patient. It is, therefore, possible and even probable that such gross outcome measures as birth weight or antenatal hospitalizations are related more to the overall milieu of the diabetic clinic than to just the medications administered. That said, the study was undertaken to compare therapies for gestational and pregestational diabetes. As the title of this report suggests, that involves comparing apples to oranges. It should be clear to the reader that gestational diabetics cannot be compared to pregestational diabetics, so throughout this report the management of these entities has been separated, even though the patients are seen in the same clinic by the same personnel. Instead we compared therapies within each category.

Several recent studies have focused on the management of gestational diabetes. Crowther and colleagues [6] randomized 1000 women with gestational diabetes to an intervention group (aggressive attempts to achieve euglycemia using insulin, not glyburide) or a routine-care group (in which caregivers were unaware of the diagnosis of glucose intolerance of pregnancy). The conclusion of that study seems obvious: "Treatment of gestational diabetes reduces serious perinatal morbidity and may also improve the woman's health-related quality of life." We agree; all women at our center who are diagnosed with gestational diabetes are transferred to our diabetic clinic for aggressive management to achieve euglycemia. The difference is that in many cases we start with oral hypoglycemic agents instead of insulin only.

Landon and co-authors [7] performed a similar trial involving 958 women. Treatment of mild gestational diabetes reduced the risks of fetal overgrowth, shoulder dystocia, cesarean delivery and hypertensive disorders compared to a group managed with usual prenatal care. The HAPO trial [8] addressed the intriguing question of whether maternal glucose levels below those diagnostic of diabetes may be associated with increased birth weight and, in fact, they were. The conclusion from these three studies [6]-[8] is that even mild gestational diabetes should be treated. The question is: how? Oral metformin [9] was tried in a recent randomized trial of 751 women. Women preferred metformin over insulin. We have not used metformin as oral therapy. Currently, the oral agent of choice is glyburide [3] [10] and we use it preferentially when fasting glucose is elevated. For postprandial hyperglycemia, we use acarbose, a little-used drug in the class of alpha-glucosidase inhibitors (enzymes in the intestines that digest carbohydrates). Because it does not affect insulin production, it does not cause hypoglycemia [11]. We have not had patients complain of gastrointestinal adverse effects. We do acknowledge that the reported experience with this drug in pregnancy is very limited. In fact, our experience reported here may be one of the largest.

Shifting now to pregestational diabetes, the focus in our report is on the use of the insulin pump. One review [12] concluded that insulin pumps may represent a superior mode of insulin delivery for many women with diabetes in pregnancy. Two recent small studies [13] [14] reported on the use of the insulin pump in pregnancy in 25 and 29 women, respectively. Results of pump use compared favorably to those achieved with multiple daily injections of insulin. We reached a similar conclusion in our study, with nearly the same number of pregnant pump patients as in these two studies [13] [14]. The methodology used in all three reports (ours and the two cited) was retrospective. Prospective randomized studies, while ideal, will be difficult to conduct due to the requirement that pump patients be both motivated and knowledgeable. We have used sensor-augmented insulin pumps on some pregnant women. A study on nonpregnant adults and children showed significant improvement in glycated hemoglobin levels associated with the use of these newer pumps [15].

Our investigation has several limitations. First and foremost is the retrospective methodology employed. Chart reviews invariably reveal missing data on many patients. We used hemoglobin A1c as an indicator of glycemic control instead of mean daily glucose, but we did not standardize the timing of the hemoglobin A1c blood draws. Selecting patients for a particular therapy was not standardized either, causing the potential for bias. Additionally, if patients were changed from oral agents to insulin, they were omitted from the study. Patients with gestational diabetes begun on split-mixed insulin initially very likely had more abnormal glucose levels and that may explain the more frequent hospitalization.

\section{Conclusion}

The presence of diabetes during pregnancy represents a significant health risk to both mother and fetus. Recog- 
nizing the different health risks associated with pregestational versus gestational diabetics, and the proper treatment options available for each, can mitigate the effects of the disease on pregnancy outcomes.

\section{Acknowledgements}

Presented in part at the first annual meeting of District XI of the American College of Obstetrics and Gynecology, we would like to thank the Department of Obstetrics and Gynecology, Texas Tech University Health Sciences Center for their support of this work.

\section{References}

[1] Chu, S.Y., Bachman, D.J., Callaghan, W.M., Whitlock, E.P., Dietz, P.M., Berg, C.J., et al. (2008) Association between Obesity during Pregnancy and Increased Use of Health Care. The New England Journal of Medicine, 358, 1444-1453. http://dx.doi.org/10.1056/NEJMoa0706786

[2] Narayan, K.M.V., Boyle, J.P., Thompson, T.J., Sorensen, S.W. and Williamson, D.F. (2003) Lifetime Risk for Diabetes Mellitus in the United States. JAMA, 290, 1884-1890. http://dx.doi.org/10.1001/jama.290.14.1884

[3] Langer, O., Conway, D.L., Berkus, M.D., Xenakis, E.M.J. and Gonzales, O. (2000) A Comparison of Glyburide and Insulin in Women with Gestational Diabetes Mellitus. The New England Journal of Medicine, 343, 1134-1138. http://dx.doi.org/10.1056/NEJM200010193431601

[4] Nicholson, W., Bolen, S., Takacs Witkop, C., Neale, D., Wilson, L. and Bass, E. (2009) Benefits and Risks of Oral Diabetes Agents Compared with Insulin in Women with Gestational Diabetes. Obstetrics \& Gynecology, 113, $193-205$. http://dx.doi.org/10.1097/AOG.0b013e318190a459

[5] Carpenter, M.W. and Coustan, D.R. (1982) Criteria for Screening Tests for Gestational Diabetes. American Journal of Obstetrics and Gynecology, 144, 768.

[6] Crowther, C.A., Hiller, J.E., Moss, J.R., McPhee, A.J., Jeffries, W.S. and Robinson, J.S. (2005) Effect of Treatment of Gestational Diabetes Mellitus on Pregnancy Outcomes. The New England Journal of Medicine, 352, 2477-2486. http://dx.doi.org/10.1056/NEJMoa042973

[7] Landon, M.B., Spong, C.Y., Thom, E., Carpenter, M.W., Ramin, S.M., Casey, B., et al. (2009) A Multicenter, Randomized Trial of Treatment for Mild Gestational Diabetes. The New England Journal of Medicine, 361, 1339-1348. http://dx.doi.org/10.1056/NEJMoa0902430

[8] The HAPO Study Cooperative Research Group (2008) Hyperglycemia and Adverse Pregnancy Outcomes. The New England Journal of Medicine, 358, 1991-2002. http://dx.doi.org/10.1056/NEJMoa0707943

[9] Rowan, J.A., Hague, W.M., Gao, W., Battin, M.R. and Moore, M.P. (2008) Metformin versus Insulin for the Treatment of Gestational Diabetes. The New England Journal of Medicine, 358, 2003-2015. http://dx.doi.org/10.1056/NEJMoa0707193

[10] Jacobson, G.F., Ramos, G.A., Ching, J.Y., Kirby, R.S., Ferrara, A. and Field, D.R. (2005) Comparison of Glyburide and Insulin for the Management of Gestational Diabetes in a Large Managed Care Organization. American Journal of Obstetrics and Gynecology, 193, 118-124. http://dx.doi.org/10.1016/j.ajog.2005.03.018

[11] Tran, N.D., Hunter, S.K. and Yankowitz, J. (2004) Oral Hypoglycemic Agents in Pregnancy. Obstetrical \& Gynecological Survey, 59, 456-463. http://dx.doi.org/10.1097/00006254-200406000-00024

[12] Wollitzer, A.D., Zisser, B.A.H. and Jovanovic, L. (2010) Insulin Pumps and Their Use in Pregnancy. Diabetes Technology \& Therapeutics, 12, S33-S36. http://dx.doi.org/10.1089/dia.2009.0187

[13] Lapolla, A., Masin, M., Bruttomesso, D., Piva, I., Crepaldi, C., Tortul, C., et al. (2003) Analysis of Outcome of Pregnancy in Type 1 Diabetics Treated with Insulin Pump or Conventional Insulin Therapy. Acta Diabetologica, 40, 143-149. http://dx.doi.org/10.1007/s00592-003-0103-1

[14] Gimenez, M., Conget, I., Nicolau, J., Pericot, A. and Levy, I. (2007) Outcome of Pregnancy in Women with Type 1 Diabetes Intensively Treated with Continuous Subcutaneous Insulin Infusion or Conventional Therapy. A Case-Control Study. Acta Diabetologica, 44, 34-37. http://dx.doi.org/10.1007/s00592-007-0239-5

[15] Bergenstal, R.M., Tamborlane, W.V., Ahmann, A., Buse, J.B., Dailey, G., Davis, S.N., et al. (2010) Effectiveness of SensorAugmented Insulin-Pump Therapy in Type 1 Diabetes. The New England Journal of Medicine, 363, 311-320. http://dx.doi.org/10.1056/NEJMoa1002853 
Scientific Research Publishing (SCIRP) is one of the largest Open Access journal publishers. It is currently publishing more than 200 open access, online, peer-reviewed journals covering a wide range of academic disciplines. SCIRP serves the worldwide academic communities and contributes to the progress and application of science with its publication.

Other selected journals from SCIRP are listed as below. Submit your manuscript to us via either submit@scirp.org or Online Submission Portal.
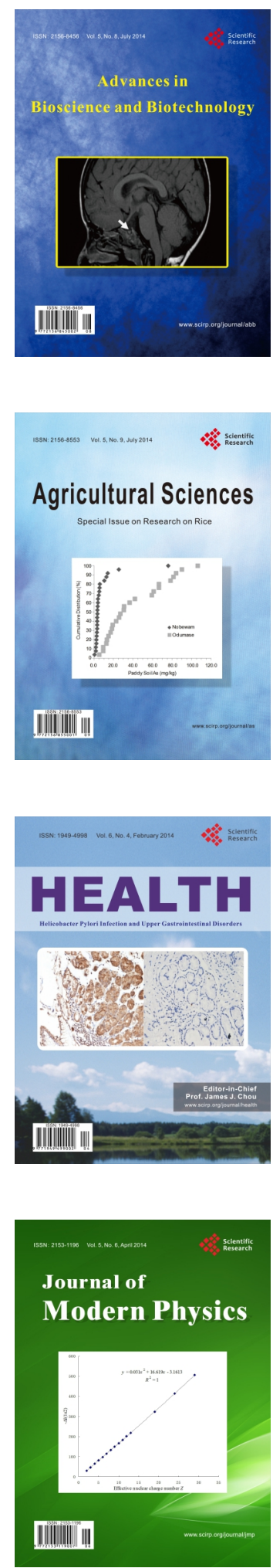
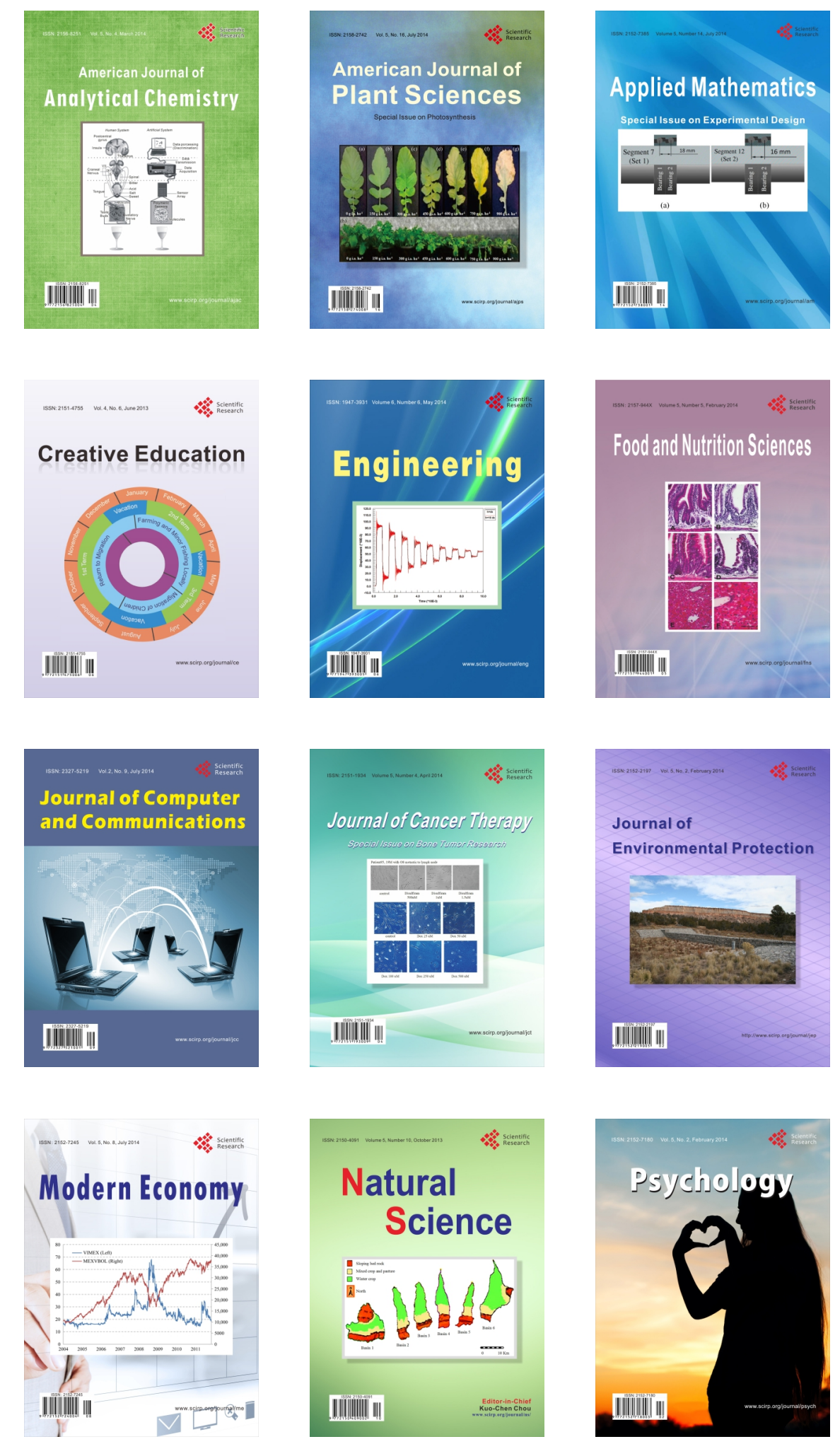